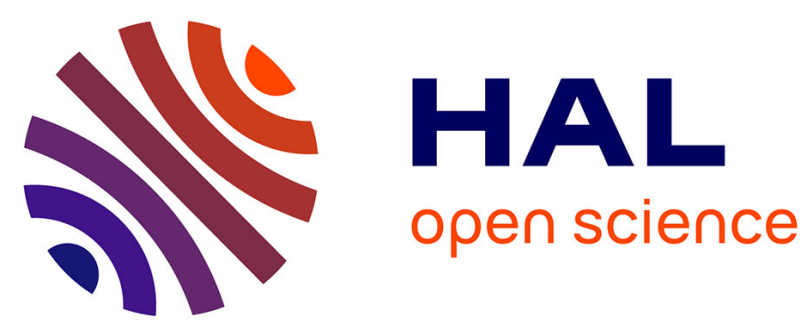

\title{
A simplified model for bi-component droplet heating and evaporation
}

Sergei S. Sazhin, E Elwardany, P.A. Krutitskii, Guillaume Castanet, Fabrice Lemoine, Elena M. Sazhina, Morgan R. Heikal

\section{- To cite this version:}

Sergei S. Sazhin, E Elwardany, P.A. Krutitskii, Guillaume Castanet, Fabrice Lemoine, et al.. A simplified model for bi-component droplet heating and evaporation. International Journal of Heat and Mass Transfer, 2010, 53, pp.4495 - 4505. 10.1016/j.ijheatmasstransfer.2010.06.044 . hal-01570433

\section{HAL Id: hal-01570433 \\ https://hal.univ-lorraine.fr/hal-01570433}

Submitted on 30 Jul 2017

HAL is a multi-disciplinary open access archive for the deposit and dissemination of scientific research documents, whether they are published or not. The documents may come from teaching and research institutions in France or abroad, or from public or private research centers.
L'archive ouverte pluridisciplinaire HAL, est destinée au dépôt et à la diffusion de documents scientifiques de niveau recherche, publiés ou non, émanant des établissements d'enseignement et de recherche français ou étrangers, des laboratoires publics ou privés. 


\title{
A simplified model for bi-component droplet heating and evaporation
}

\author{
S.S. Sazhin ${ }^{\mathrm{a}, *}$, A. Elwardany ${ }^{\mathrm{a}}$, P.A. Krutitskii ${ }^{\mathrm{b}}$, G. Castanet $^{\mathrm{c}}$, F. Lemoine ${ }^{\mathrm{c}}$, E.M. Sazhina ${ }^{\text {a }}$, M.R. Heikal ${ }^{\text {a }}$ \\ a Sir Harry Ricardo Laboratories, Centre for Automotive Engineering, School of Environment and Technology, Faculty of Science and Engineering, \\ University of Brighton, Brighton BN2 4GJ, UK \\ ${ }^{\mathrm{b}}$ Keldysh Institute for Applied Mathematics, Department 4, Miusskaya Sq. 4, Moscow 125047, Russia \\ ${ }^{\mathrm{c}}$ LEMTA, Nancy-Université, CNRS UMR 7563, 2, Avenue de la Forêt de Haye, BP 160, 54504 Vanduvre-lès-Nancy, France
}

\section{Keywords:}

Droplets

Multi-component fuel

Heating

Evaporation

Diffusion equation

Acetone

Ethanol

\begin{abstract}
A B S T R A C T
A simplified model for bi-component droplet heating and evaporation is developed and applied for the analysis of the observed average droplet temperatures in a monodisperse spray. The model takes into account all key processes, which take place during this heating and evaporation, including the distribution of temperature and diffusion of liquid species inside the droplet and the effects of the non-unity activity coefficient (ideal and non-ideal models). The effects of recirculation in the moving droplets on heat and mass diffusion within them are taken into account using the effective thermal conductivity and the effective diffusivity models. The previously obtained analytical solution of the transient heat conduction equation inside droplets is incorporated in the numerical code alongside the original analytical solution of the species diffusion equation inside droplets. The predicted time evolution of the average temperatures is shown to be reasonably close to the measured one, especially in the case of pure acetone and acetone-rich mixture droplets. It is shown that the temperatures predicted by the simplified model and the earlier reported vortex model are reasonably close. Also, the temperatures predicted by the ideal and non-ideal models differ by not more than several degrees. This can justify the application of the simplified model with the activity coefficient equal to 1 for the interpretation of the time evolution of temperatures measured with errors more than several degrees.
\end{abstract}

\section{Introduction}

In a number of papers a new model for heating and evaporation of monocomponent droplets, based on the analytical solution of the heat conduction equation inside droplets, has been described $[1-3]$. The effects described by this model were shown to be particularly important for modelling fuel spray autoignition processes $[4,5]$. It was shown to be more CPU efficient compared with the model based on the numerical solution of the heat conduction equation inside droplets [6], and was validated against the results of available experimental measurements of droplet temperatures $[7,8]$.

The practical application of the above-mentioned model, however, is limited by the fact that most real-life fuels are multi-component. The models of multi-component droplet heating and evaporation could be subdivided into two main groups: those based on the analysis of individual components [9-15], applicable in the case when a small number of components needs to be taken into account, and those based on the probabilistic analysis of a large number of components (e.g. continuous thermodynamics ap-

\footnotetext{
* Corresponding author. Tel.: +44 (0)1273642677; fax: +44 (0)1273642309.

E-mail address: S.Sazhin@brighton.ac.uk (S.S. Sazhin).
}

proach [16-18] and the Distillation Curve Model [19]). In the second family of models a number of additional simplifying assumptions were used, including the assumption that species inside droplets mix infinitely quickly. A model containing features of both these groups of models has been suggested in [20].

The focus of this paper is on the extension of the model developed in $[2,3]$ for monocomponent droplets to the case of multicomponent droplets. Only the first group of multi-component droplet heating and evaporation models is considered, which allows us to perform the deterministic analysis of individual species. Moreover, at this stage only the simplest case of bi-component droplets is considered, as in $[12,13,15]$, which allows us to develop a better understanding of the underlying physics of the processes involved. The new model is much simpler than the previously suggested models (e.g. [15]) which makes it potentially attractive for implementation into computational fluid dynamics (CFD) codes. The predictions of the model are compared with the measured time evolution of droplet temperatures in monodisperse bi-component (ethanol/acetone) droplet streams.

The basic equations and approximations of the new model are described in Section 2. The experimental set-up for measurements of droplet temperatures in monodisperse bi-component (ethanol/ acetone) droplet streams is briefly described in Section 3. In 


\section{Nomenclature}

$B_{M} \quad$ Spalding mass transfer number

$B_{T} \quad$ Spalding heat transfer number

C specific heat capacity

C distance parameter

D diffusion coefficient

$F \quad$ function introduced in Eq. (8) or (22)

$G^{E} \quad$ Gibbs free energy per unit mole

$h \quad$ convection heat transfer coefficient

$h_{0} \quad \frac{h}{k_{l}} R_{d}-1$ or $-\frac{\alpha}{D_{l}} R_{d}-1$

$k$ thermal conductivity

$k_{B} \quad$ Boltzmann constant

$L \quad$ latent heat of evaporation

m mass

M molar mass

$n \quad$ molar concentration

$\mathrm{Nu} \quad$ Nusselt number

$p \quad$ pressure

Pe Peclet number

$\mathrm{Pr} \quad$ Prandtl number

$q_{n} \quad$ parameter introduced in Eq. (3)

$Q_{I} \quad$ power spent on droplet heating

$Q_{n} \quad$ parameter introduced by Eq. (14)

$R$ distance from the droplet centre

$R_{u} \quad$ universal gas constant

$R_{d} \quad$ droplet radius

$\mathrm{Re} \quad$ Reynolds number

Sc Schmidt number

Sh Sherwood number

$t$ time

$T \quad$ temperature

$\widetilde{T}_{0}(R) \quad R T_{d 0}(R)$

$v_{A} \quad$ molar volume

$X \quad$ molar fraction

$Y \quad$ mass fraction

Greek symbols

$\alpha \quad$ parameter defined by Eq. (11) or introduced in Eq. (44)

Section 4, some results of the numerical solution of the basic equations for the values of parameters relevant to the experimental setup described in Section 3 are presented. The main results of the paper are summarised in Section 5.

\section{Basic equations and approximations}

The model developed in the paper is based on the equations describing, liquid phase heating, species diffusion in the liquid phase, droplet evaporation, and species mass fractions at the surface of the droplets. These equations and their approximations and analytical solutions, where appropriate, are presented and discussed below.

\subsection{Droplet heating}

As in the case of monocomponent droplets [1-3], the process of heating (or cooling) for stationary spherically-symmetric multicomponent droplets is described by the following transient heat conduction equation for the temperature $T \equiv T(t, R)$ in the liquid phase [21,22]:

$\frac{\partial T}{\partial t}=\kappa\left(\frac{\partial^{2} T}{\partial R^{2}}+\frac{2}{R} \frac{\partial T}{\partial R}\right)$, $\beta \quad$ parameter introduced in Eq. (44)

$\delta \quad$ parameter introduced in Eq. (44)

$\gamma \quad$ activity coefficient

$\epsilon \quad$ evaporation rate

$\varepsilon_{v, a} \quad$ Lennard-Jones energy

$\eta \quad$ function defined by Eq. (28)

$\kappa \quad$ thermal diffusivity

$\kappa_{R} \quad$ parameter introduced in Eq. (3)

$\lambda, \lambda_{n} \quad$ eigenvalues

$\mu \quad$ dynamic viscosity

$\mu_{0}(t) \quad$ variable introduced in Eq. (3)

$v \quad$ kinematic viscosity

$\rho \quad$ density

$\sigma \quad$ Lennard-Jones length

$\varphi \quad$ parameter defined by Eq. (24) or associated factor of solvent

$\chi \quad$ parameter defined by Eq. (7)

$\chi_{Y} \quad$ parameter defined by Eq. (18)

Subscripts

ac acetone

amb ambient

b boiling

d droplet

eff effective

eth ethanol

g gas

$i \quad$ species

iso isolated

$l \quad$ liquid

$p \quad$ constant pressure

$s \quad$ surface

$v \quad$ vapour

$0 \quad$ initial

$\infty \quad$ ambient conditions where $\kappa=k_{l} /\left(c_{l} \rho_{l}\right)$ is the liquid thermal diffusivity, $k_{l}, c_{l}$, and $\rho_{l}$ are the liquid thermal conductivity, specific heat capacity, and density, respectively, $R$ is the distance from the centre of the spherical droplet, $t$ is time.

Eq. (1) inside the droplet is to be solved for $t>0$ and $0 \leqslant R<R_{d}$, where $R_{d}$ is the droplet radius, with the following boundary condition:

$h\left(T_{g}-T_{s}\right)=\left.k_{l} \frac{\partial T}{\partial R}\right|_{R=R_{d}-0}$

and the initial condition $T(t=0)=T_{d 0}(R)$, where $T_{s}=T_{s}(t)$ is the droplet's surface temperature, $T_{g}=T_{g}(t)$ is the ambient gas temperature, $h$ is the convection heat transfer coefficient, linked with the Nusselt number $\mathrm{Nu}$ via the following equation:

$\mathrm{Nu}=2 R_{d} h / k_{g}$

where $k_{\mathrm{g}}$ is the gas thermal conductivity.

Remembering the physical background to the problem, we are interested only in a solution which is continuously differentiable twice in the whole domain. This implies that $T$ should be bounded for $0 \leqslant R<R_{d}$.

Assuming that $h=$ const., the solution of Eq. (1), subject to the above boundary and initial conditions, can be presented as [2]: 


$$
\begin{aligned}
T(R, t)= & \frac{1}{R} \sum_{n=1}^{\infty}\left\{q_{n} \exp \left[-\kappa_{R} \lambda_{n}^{2} t\right]-\frac{R_{d}^{2} \sin \lambda_{n}}{\left\|v_{n}\right\|^{2} \lambda_{n}^{2}}\left(\mu_{0}(0)-\frac{\mu_{0}^{\prime}}{\kappa_{R} \lambda_{n}^{2}}\right)\right. \\
& \left.\times \exp \left[-\kappa_{R} \lambda_{n}^{2} t\right]-\frac{R_{d}^{2} \sin \lambda_{n}}{\left\|v_{n}\right\|^{2} \lambda_{n}^{4}} \frac{\mu_{0}^{\prime}}{\kappa_{R}}\right\} \sin \left[\lambda_{n}\left(\frac{R}{R_{d}}\right)\right]+T_{g}(t),
\end{aligned}
$$

where $\lambda_{n}$ are solutions of the equation:

$\lambda \cos \lambda+h_{0} \sin \lambda=0$,

$\left\|v_{n}\right\|^{2}=\frac{R_{d}}{2}\left(1-\frac{\sin 2 \lambda_{n}}{2 \lambda_{n}}\right)=\frac{R_{d}}{2}\left(1+\frac{h_{0}}{h_{0}^{2}+\lambda_{n}^{2}}\right)$,

$q_{n}=\frac{1}{\left\|v_{n}\right\|^{2}} \int_{0}^{R_{d}} \widetilde{T}_{0}(R) \sin \left[\lambda_{n}\left(\frac{R}{R_{d}}\right)\right] \mathrm{d} R$,

$\kappa_{R}=\frac{k_{l}}{c_{l} \rho_{l} R_{d}^{2}}, \quad \mu_{0}(t)=\frac{h T_{g}(t) R_{d}}{k_{l}}$,

$h_{0}=\left(h R_{d} / k_{l}\right)-1, \widetilde{T}_{0}(R)=R T_{d 0}(R)$. The solution of Eq. (4) gives a set of positive eigenvalues $\lambda_{n}$ numbered in ascending order $(n=1,2, \ldots)$. The trivial solution $\lambda=0$ is not considered.

Note that the norm $\left\|v_{n}\right\|^{2}$ differs from the norm chosen in [2] by the factor $R_{d}$ (see their Eq. (A4)). This does not affect the final solution.

Solution (3) is valid for $h_{0}>-1$, which is satisfied, remembering the physical background of the problem $(h>0)$. The condition $h=$ const. is valid for sufficiently small time steps.

When deriving Solution (3) it was assume that it is applied to individual short time steps. In this case we can ignore the time dependence of $\frac{\mathrm{d} \mu_{0}(t)}{\mathrm{d} t}$ during the time steps and assume that $\frac{\mathrm{d} \mu_{0}(t)}{\mathrm{d} t} \approx\left\langle\frac{\mathrm{d} \mu_{0}(t)}{\mathrm{d} t}\right\rangle \equiv \mu_{0}^{\prime}$. Note that $\mu_{0}$ depends on time via $T_{g}$ only.

To take into account the effect of droplet evaporation in analytical solution (3), gas temperature should be replaced by the socalled effective temperature defined as:

$T_{\text {eff }}=T_{g}+\frac{\rho_{l} L \dot{R}_{d}}{h}$

where $L$ is the latent heat of evaporation, the value of $\dot{R}_{d}$ can be taken from the previous time step and estimated based on Eq. (27). $R_{d}$ is assumed constant in the analytical solutions, but is updated at the end of the time step $\Delta t$ :

$R_{d(\text { new })}=R_{d(\text { old })}+\dot{R}_{d} \Delta t$,

where the value of $\dot{R}_{d}$ is controlled by the droplet evaporation model (see Eq. (27) in Section 2.3). Note that updating of liquid density can lead to $R_{d(\text { new })}>R_{d \text { (old) }}$ (thermal swelling, see Eq. (27)).

The average surface temperature in a moving droplet can still be correctly predicted by Eq. (1), with appropriate boundary conditions, and its solution ( 3 ) if the liquid thermal conductivity $k_{l}$ is replaced by the so-called effective thermal conductivity $k_{\text {eff }}[23]$ :

$k_{\text {eff }}=\chi k_{l}$,

where the coefficient $\chi$ varies from 1 (at droplet Peclet number $\mathrm{Pe}_{d(-}$ $\left.\left.{ }_{l}\right)=\operatorname{Re}_{d(l)} \operatorname{Pr}_{l}<10\right)$ to $2.72\left(\right.$ at $\left.\mathrm{Pe}_{d(l)}>500\right)$ and can be approximated as:

$\chi=1.86+0.86 \tanh \left[2.225 \log _{10}\left(\mathrm{Pe}_{d(l)} / 30\right)\right]$.

Liquid fuel transport properties were used for calculating $\mathrm{Pe}_{d(l)}$. The model based on Eqs. (6) and (7) is known as the effective thermal conductivity model. This model was developed mainly for the estimation of the average surface temperature of droplets, which controls droplet evaporation (cf. [24,25]). It cannot predict adequately the details of the distribution of temperature inside droplets, which include vortex structures for non-zero droplet velocities, but these are not required in most practical engineering applications. Hence, the applicability of this model can be justified. It allows the applica- tion of Solution (3) to the case of moving droplets by replacing $k_{l}$ with $k_{\text {eff }}$, assuming that $h=$ const.

The value of $\mathrm{Nu}$ for an isolated moving droplet is estimated based on the following equation [23]:

$$
\begin{aligned}
\mathrm{Nu}_{\text {iso }}= & 2 \frac{\ln \left(1+B_{T}\right)}{B_{T}} \\
& \times\left(1+\frac{\left(1+\operatorname{Re}_{d} \operatorname{Pr}_{d}\right)^{1 / 3} \max \left[1, \operatorname{Re}_{d}^{0.077}\right]-1}{2 F\left(B_{T}\right)}\right),
\end{aligned}
$$

where

$B_{T}=\frac{c_{p v}\left(T_{g}-T_{s}\right)}{L_{\mathrm{eff}}}$

is the Spalding heat transfer number,

$F\left(B_{T}\right)=\left(1+B_{T}\right)^{0.7} \frac{\ln \left(1+B_{T}\right)}{B_{T}}$,

$L_{\text {eff }}=L+\frac{Q_{L}}{\dot{m}_{d}}=\sum_{i} \epsilon_{i} L_{i}+\frac{Q_{L}}{\sum_{i} \dot{m}_{i}}, Q_{L}$ is the power spent on droplet heating, $c_{p v}$ is the specific heat capacity of fuel vapour, $\epsilon_{i}=\epsilon_{i}(t)$ are the evaporation rates of species $i, \dot{m}_{i}=\epsilon_{i} \dot{m}_{d}\left(\dot{m}_{d}=\sum_{i} \dot{m}_{i}\right)$.

This model is equally applicable to one-component and multicomponent droplets. The liquid thermal conductivity of multicomponent droplets is calculated as described in Appendix A.

\subsection{Species diffusion in the liquid phase}

Assuming that the processes inside droplets are spherically symmetric (no convection), equations for mass fractions of liquid species $Y_{l i} \equiv Y_{l i}(t, R)$ there can be presented in the following form:

$\frac{\partial Y_{l i}}{\partial t}=D_{l}\left(\frac{\partial^{2} Y_{l i}}{\partial R^{2}}+\frac{2}{R} \frac{\partial Y_{l i}}{\partial R}\right)$,

where $i=1,2, D_{l}$ is the liquid mass diffusivity.

Eq. (9) is solved with the following boundary condition [26,5]:

$\alpha\left(\epsilon_{i}-Y_{\text {lis }}\right)=-\left.D_{l} \frac{\partial Y_{l i}}{\partial R}\right|_{R=R_{d}-0}$,

and the initial condition $Y_{l i}(t=0)=Y_{l i 0}(R)$, where $Y_{l i s}=Y_{l i s}(t)$ are liquid components' mass fractions at the droplet's surface,

$\alpha=\frac{\left|\dot{m}_{d}\right|}{4 \pi \rho_{l} R_{d}^{2}}$,

$\dot{m}_{d}$ is the droplet evaporation rate, the calculation of which is discussed in the next section (see Eq. (20)).

Assuming that species concentrations in the ambient gas are equal to zero, the values of $\epsilon_{i}$ can be found from the following relation [9-11]:

$\epsilon_{i}=\frac{Y_{v i s}}{\sum_{i} Y_{v i s}}$,

where the subscript $v$ indicates the vapour phase. We assume that $\epsilon_{i}$ is constant and is determined by the values of $Y_{v j s}(j$ refers to all species) at the beginning of the time step. The conditions $\epsilon_{i}=$ const. and $\alpha=$ const. can always be guaranteed for sufficiently small time steps.

As in the case of Eq. (1), we are interested only in a solution which is continuously differentiable twice in the whole domain. This implies that $Y_{l i}$ should be bounded for $0 \leqslant R<R_{d}$. Moreover, the physical meaning of $Y_{l i}$, as the mass fraction, implies that $0 \leqslant Y_{l i} \leqslant 1$.

Eq. (9) with boundary conditions (10) have essentially the same structure as Eq. (1) with boundary condition (2). The former equations can be obtained from the latter if we replace $T$ with $Y_{l i}, \kappa$ with 
$D_{l}, T_{g}$ with $\epsilon_{i}, T_{s}$ with $Y_{l i s}$ and $k_{l} / h$ with $-D_{l} / \alpha$. However, although Eq. (9) look rather similar to Eq. (1), the solution of Eq. (1) (see Expression (3)) cannot be used for (9). The reason for this is that solution (3) is valid only for $h_{0}>-1$. At the same time the boundary condition for Eq. (9) at $R=R_{d}$ (see Eq. (10)) leads to the situation in which $h_{0}<-1$. The solution of Eq. (9) in this case can be presented as

$$
\begin{aligned}
Y_{l i}= & \epsilon_{i}+\frac{1}{R}\left\{\left[\exp \left[D_{l}\left(\frac{\lambda_{0}}{R_{d}}\right)^{2} t\right]\left[q_{i 0}-Q_{0} \epsilon_{i}\right] \sinh \left(\lambda_{0} \frac{R}{R_{d}}\right)\right.\right. \\
& \left.+\sum_{n=1}^{\infty}\left[\exp \left[-D_{l}\left(\frac{\lambda_{n}}{R_{d}}\right)^{2} t\right]\left[q_{i n}-Q_{n} \epsilon_{i}\right] \sin \left(\lambda_{n} \frac{R}{R_{d}}\right)\right]\right\},
\end{aligned}
$$

where $\lambda_{0}$ and $\lambda_{n}(n \geqslant 1)$ are solutions of equations

$\tanh \lambda=-\frac{\lambda}{h_{0}}$ and $\tan \lambda=-\frac{\lambda}{h_{0}}$.

respectively, $h_{0}=-\left(1+\frac{\alpha R_{d}}{D_{l}}\right)$.

$Q_{n}= \begin{cases}-\frac{1}{\left\|v_{0}\right\|^{2}}\left(\frac{R_{d}}{\lambda_{0}}\right)^{2}\left(1+h_{0}\right) \sinh \lambda_{0} & \text { when } n=0, \\ \frac{1}{\left\|v_{n}\right\|^{2}}\left(\frac{R_{d}}{\lambda_{n}}\right)^{2}\left(1+h_{0}\right) \sin \lambda_{n} & \text { when } n \geqslant 1,\end{cases}$

$q_{\text {in }}=\frac{1}{\left\|v_{n}\right\|^{2}} \int_{0}^{R_{d}} R Y_{l i 0}(R) v_{n}(R) \mathrm{d} R$,

$n \geqslant 0$, where

$v_{0}(R)=\sinh \left(\lambda_{0} \frac{R}{R_{d}}\right), \quad v_{n}(R)=\sin \left(\lambda_{n} \frac{R}{R_{d}}\right), n \geqslant 1$.

If Eq. (13) is applied to a short time step it can be simplified to:

$$
\begin{aligned}
Y_{l i}= & \epsilon_{i}+\frac{1}{R}\left\{\left[1+D_{l}\left(\frac{\lambda_{0}}{R_{d}}\right)^{2} t\right]\left[q_{i 0}-Q_{0} \epsilon_{i}\right] \sinh \left(\lambda_{0} \frac{R}{R_{d}}\right)\right. \\
& \left.+\sum_{n=1}^{\infty}\left[1-D_{l}\left(\frac{\lambda_{n}}{R_{d}}\right)^{2} t\right]\left[q_{i n}-Q_{n} \epsilon_{i}\right] \sin \left(\lambda_{n} \frac{R}{R_{d}}\right)\right\} .
\end{aligned}
$$

The average mass fraction of species in a moving droplet can still be correctly predicted by Eq. (9), with appropriate boundary conditions, if the liquid diffusivity $D_{l}$ is replaced with the so-called effective diffusivity $D_{\text {eff }}[27,26]$ :

$D_{\text {eff }}=\chi_{Y} D_{l}$,

where the coefficient $\chi_{Y}$ varies from 1 to 2.72 and can be approximated as:

$\chi_{Y}=1.86+0.86 \tanh \left[2.225 \log _{10}\left(\operatorname{Re}_{l} \mathrm{Sc}_{l} / 30\right)\right]$,

$\mathrm{Sc}_{l}$ is the liquid Schmidt number defined as:

$\mathrm{Sc}_{l}=\frac{v_{l}}{D_{l}}$

$v_{l}$ is the liquid kinematic viscosity.

Eq. (17) allows the application of Solution (13) to the case of

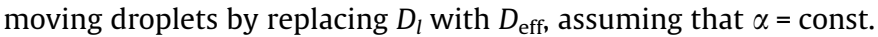
Following [26] this model is called the effective diffusivity model. As in the case of the effective thermal conductivity model, this model cannot describe the details of species mass fractions inside droplets, including vortex structures in the moving droplets, but this information is not required in most engineering applications. A more complex approach based on the analysis of Hill vortices is discussed in a number of publications including [26,15].

The liquid kinematic viscosity and diffusivity in multi-component droplets are calculated as described in Appendix A.

\subsection{Droplet evaporation}

In the case of isolated moving monocomponent droplets, their evaporation rate is given by the following equation [5]:

$\dot{m}_{d}=-2 \pi R_{d} D_{v} \rho_{\text {total }} B_{M} \mathrm{Sh}_{\text {iso }}$,

where $D_{v}$ is the binary diffusion coefficient of vapour in air and $B_{M}$ is the Spalding mass transfer number defined as:

$B_{M}=\frac{\rho_{v s}-\rho_{v \infty}}{\rho_{g s}}=\frac{Y_{v s}-Y_{v \infty}}{1-Y_{v s}}$,

$Y_{v}$ is the vapour mass fraction and $\mathrm{Sh}_{\text {iso }}$ is the Sherwood number approximated for the isolated droplets by the following expression:

$\mathrm{Sh}_{\mathrm{iso}}=2 \frac{\ln \left(1+B_{M}\right)}{B_{M}}\left(1+\frac{\left(1+\operatorname{Re}_{d} \mathrm{Sc}_{d}\right)^{1 / 3} \max \left[1, \mathrm{Re}_{d}^{0.077}\right]-1}{2 F\left(B_{M}\right)}\right)$

$\mathrm{Sc}_{d}=\frac{v_{\text {air }}}{D_{v}}$

is the Schmidt number,

$F\left(B_{M}\right)=\left(1+B_{M}\right)^{0.7} \frac{\ln \left(1+B_{M}\right)}{B_{M}}$.

$B_{T}$ and $B_{M}$ are linked by the following equation [23]:

$B_{T}=\left(1+B_{M}\right)^{\varphi}-1$,

where

$\varphi=\left(\frac{c_{p v}}{c_{p, \text { air }}}\right) \frac{1}{\mathrm{Le}}$,

Le $=k_{g} /\left(c_{p, \text { air }} \rho_{\text {total }} D_{v}\right)$ is the Lewis number.

In the case of multi-component droplets the problem of modelling droplet evaporation is complicated by the fact that different species diffuse at different rates, and the evaporation rate of one of the species is affected by the evaporation rate of other species. In [15] the analysis of evaporation of multi-component droplets led to the following expression for $\dot{m}_{d}$ :

$\dot{m}_{d}=-2 \pi R_{d} D_{i v} \rho_{\text {total }} B_{M i} S_{\text {iso }(i)}$,

where $B_{M i}$ is the species Spalding mass transfer number defined as:

$B_{M i}=\frac{Y_{i v s}-Y_{i v \infty}}{\epsilon_{i}-Y_{i v s}}$,

$D_{i v}$ is the diffusion coefficient of species $i$ in air, $S h_{\text {iso }(i)}$ is defined by (22) replacing $B_{M}$ by $B_{M i}$ and $D_{v}$ by $D_{i v}$. Remembering (12) one can see that $B_{M}=B_{M i}$. Hence, for stationary droplets this leads to the paradox that the same value of $\dot{m}_{d}$ is predicted by Eq. (25) for different $D_{i v}$. This paradox is resolved by the fact that although Eq. (25) is correct, the value of $\mathrm{Sh}_{\text {iso(i) }}$ cannot be approximated by the analogue of Eq. (22) which is implicitly based on the assumption that the evaporating species do not affect each other.

Our analysis of $\dot{m}_{d}$ is based on Eq. (20), assuming that the mixture of vapour species can be treated as a separate gas, similar to treating the mixture of nitrogen, oxygen and carbon dioxide as air $\left(Y_{v s}=\sum_{i} Y_{v i s}\right)$. The value of $D_{v}$ is estimated as described in Appendix A.

When calculating the value of $\dot{R}_{d}$ we took into account both droplet evaporation during the time step, and the change in their density during this time step (see Eq. (20) in [3]):

$\dot{R}_{d}=\frac{\dot{m}_{d}}{4 \pi R_{d}^{2} \rho_{l}}+\frac{R_{d}\left(\bar{T}_{0}\right)}{\Delta t}\left[\left(\frac{\rho\left(\bar{T}_{0}\right)}{\rho\left(\bar{T}_{1}\right)}\right)^{1 / 3}-1\right]$,

where $\bar{T}_{0}$ and $\bar{T}_{1}$ are average droplet temperatures at the beginning $t=t_{0}$ and the end of the time step $t=t_{1}, \Delta t=t_{1}-t_{0}$. 
The corrections to $\mathrm{Nu}_{\text {iso }}$ and $\mathrm{Sh}_{\text {iso }}$ due to the finite distance between droplets are calculated as in [7,35]:

$$
\begin{aligned}
\eta(C) & =\frac{\mathrm{Sh}}{\mathrm{Sh}_{\text {iso }}}=\frac{\mathrm{Nu}}{\mathrm{Nu}_{\text {iso }}} \\
& =1-0.57\left(1-\frac{1-0.57 \exp [-0.13(C-6)]}{1+0.57 \exp [-0.13(C-6)]}\right),
\end{aligned}
$$

where $C$ is the ratio of the distance between droplets and their diameters (distance parameter).

\subsection{Species mass fractions at the surface of the droplets}

To calculate the species mass evaporation rate $\dot{m}_{i}$ and the values of the evaporation rate of species $\epsilon_{i}$ based on Eq. (12) we need to calculate first the values of $Y_{v i s}$. The latter depends on the partial pressure of species $i$ in the vapour state in the immediate vicinity of the droplet surface [28]:

$p_{v i}=X_{l i s} \gamma_{i} p_{v i}^{*}$,

where $X_{l i s}$ is the molar fraction of the $i$ th species in the liquid near the droplet surface, $p_{v i}^{*}$ is the partial vapour pressure of the $i$ th species in the case when $X_{l i s}=1, \gamma_{i}$ is the activity coefficient. The subscript $s$ is omitted in Eq. (29) and all the following equations in this subsection.

In the limit when $\gamma_{i}=1$, Eq. (29) describes the Raoult law. A more accurate approximation for $\gamma_{i}$ for the ethanol-acetone mixture is described in Appendix B, following [29,30,15]. This approximation will be used in our analysis.

Remembering the Clausius-Clapeyron equation [28], Eq. (29) can be re-written as:

$p_{v i}=X_{l i s} \gamma_{i} p_{\text {amb }} \exp \left[\frac{L_{i} M_{i}}{R_{u}}\left(\frac{1}{T_{b i}}-\frac{1}{T_{s}}\right)\right]$

where $M_{i}$ is the molar mass and $T_{b i}$ is the boiling temperature of the $i$ th species, $p_{\mathrm{amb}}$ is the ambient pressure. When deriving Eq. (30) it was taken into account that $p_{v i}^{*}$ is equal to the ambient pressure when $T_{s}=T_{b i}, L_{i}$ is the latent heat of evaporation of species $i$. Note that there are typos in Eqs. (135) and (136) of [5].

The numerical values of $L_{i} M_{i}$ at $T=T_{b i}$, values of $T_{b i}$ for ethanol and acetone at atmospheric pressure, and $M_{i}$ are presented in Table 1 [31].

The values of $L_{i}$ for ethanol and acetone (in $\mathrm{J} / \mathrm{kg}$ ) at arbitrary $T$ are calculated based on the following equations [30,34]:

$$
\begin{aligned}
& L_{\text {eth }}=120.91 \times 10^{3}(516.2-T)^{0.38}, \\
& L_{\mathrm{ac}}=489 \times 10^{3}\left(\frac{508.1-T}{508.1-T_{b, \mathrm{ac}}}\right)^{0.38},
\end{aligned}
$$

where $T$ is in $\mathrm{K}, T_{b \text {,ac }}$ is the boiling temperature of acetone given in Table 1.

Eq. (30) can be re-written for the molar fractions of species $i$ in the vapour phase:

$X_{v i}=X_{l i s} \gamma_{i} \exp \left[\frac{L_{i} M_{i}}{R_{u}}\left(\frac{1}{T_{b i}}-\frac{1}{T_{s}}\right)\right]$.

\section{Experimental set-up and input parameters}

The experimental set-up used for validation of the model is the same as described in $[7,15,30]$. A monodisperse droplet stream was

Table 1

The values $L_{i} M_{i}$ at $T=T_{b i}, T_{b i}$ and $M_{i}$ for ethanol and acetone.

\begin{tabular}{llll}
\hline Substance & $L_{i} M_{i}(\mathrm{~kJ} / \mathrm{mol})$ & $T_{b i}(\mathrm{~K})$ & $M_{i}(\mathrm{~kg} / \mathrm{kmol})$ \\
\hline Ethanol $\left(\mathrm{C}_{2} \mathrm{H}_{6} \mathrm{O}\right)$ & 38.56 & 351.80 & 46.069 \\
Acetone $\left(\mathrm{C}_{3} \mathrm{H}_{6} \mathrm{O}\right)$ & 29.10 & 329.22 & 58.080 \\
\hline
\end{tabular}

generated by a Rayleigh-type disintegrating liquid jet. The fuel temperature was regulated in the injector body and was measured near the outlet by a thermocouple. Downstream distance from the injector was converted into time with the help of the space evolution of the droplet velocity measured using the Laser Doppler Anemometry (LDA) technique. Droplets were injected into a quiescent atmosphere at room temperature. Droplet temperatures were measured using the technique described in $[7,15,30]$. The input parameters for the model were the initial droplet temperature (assuming that this temperature is initially homogeneous), ambient gas temperature (assuming that this temperature remains constant during the experiment), the distance parameter (ratio of the distances between droplets and their diameters) and the droplet velocities.

The measured time evolution of the droplet velocities for pure acetone is shown in Fig. 1. In the same figure, the linear approximation of the experimental results is shown. Similar plots were obtained for pure ethanol and various mixtures of acetone and ethanol (not shown). In all cases the linear approximations of the experimental results, similar to the one shown in Fig. 1, were used. These are summarised in Table 2.

The two-colour laser-induced fluorescence (2cLIF) technique, previously used for the measurement of droplet temperatures, was further developed to include the third colour band. This additional band is required for the analysis of bi-component droplets [32]. In this new technique, droplets were seeded with a low concentration of a fluorescent dye and illuminated by laser beams having a wavelength tuned on the absorption spectrum of the tracer. The fluorescence signal was detected over specific spectral bands of emissions. The ratio of the intensity of these bands depends on the temperature and, to a lesser extent, on the droplet composition, while the fluorescence dependencies on tracer concentration, probe volume dimensions, laser intensity and optical layout were eliminated [32]. The probe volume (intersection between the laser beams and the detection field of view) was about $150 \times 150 \times 1200 \mu \mathrm{m}^{3}$. It was larger than the droplet in order to provide a global excitation of the whole droplet volume. The signal was averaged over the total time of droplet transit in the probe volume.

In the presence of thermal gradients inside droplets, the temperature obtained based on this signal could differ from the volume-averaged droplet temperature. This is related to the fact that the laser intensity is not distributed uniformly inside the droplet, mainly due to light refraction at the droplet surface. Also, the receiving optics collect the fluorescent signal from the droplet interior with a spatial distortion. These effects could be taken into account with the help of an efficient ray tracing code, based on the geometric optics approximation [33]. This analysis, however, is beyond the scope of this paper. We would just mention that the 2cLIF measurements allow us to estimate the volume-averaged droplet temperature with systematically more weighting in the zones near the centre of the droplet rather than at its edge. We cannot quantify this effect at this stage and we assume that the measured temperatures are close to the droplet average temperatures.

\section{Results}

The plots of the time dependence of the temperatures for pure acetone and ethanol are shown in Figs. 2 and 3, respectively. The experimentally observed average droplet temperatures are shown by filled triangles in these figures. The results of calculations are shown by solid, dashed and dashed dotted curves referring to the central, average and surface temperatures. The calculations started with the first observed droplets approximately $1 \mathrm{~ms}$ after the start of injection. At the earlier times, the liquid fuel formed an unstable 




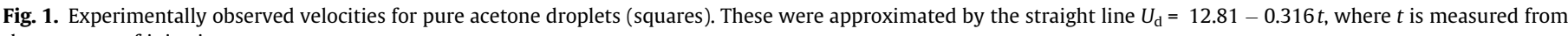
the moment of injection.

Table 2

The approximations of $U_{\text {drop }}$.

\begin{tabular}{ll}
\hline Substance & Approximation of $U_{\mathrm{drop}}$ in $\mathrm{m} / \mathrm{s}(t$ is in $\mathrm{ms})$ \\
\hline $100 \%$ acetone & $12.81-0.316 t$ \\
$100 \%$ ethanol & $12.30-0.344 t$ \\
$25 \%$ ethanol $+75 \%$ acetone & $12.75-0.370 t$ \\
$50 \%$ ethanol $+50 \%$ acetone & $12.71-0.448 t$ \\
$75 \%$ ethanol $+25 \%$ acetone & $12.28-0.306 t$ \\
\hline
\end{tabular}

jet the temperature evolution of which cannot be interpreted using our model. Also, we assumed that there is no temperature gradient inside droplets at the initial moment of time used in calculations.

As follows from these figures, all three temperatures are well separated for both acetone and ethanol. Hence, the difference between them needs to be taken into account in the analysis of experimental data (cf. $[36,37]$ ). In the case of acetone there seems to be a reasonable agreement between the values of average temperature of the droplets and experimental data. Data on the time evolution of droplet radii were not available. Note that the effective thermal conductivity and effective diffusivity models, on which our analysis is based, are primarily designed to predict correctly the average surface temperature and species mass fractions of droplets, but not their average temperature and species mass fractions.

In the case of ethanol, shown in Fig. 3, the experimentally observed temperatures lie between average and surface temperatures predicted by the model. Note that the temperature scale in Fig. 3 is much finer than in Fig. 2. Hence the overall agreement between experimental data and predictions of the model looks reasonably good. This level of agreement between the model and experimental data is similar to the one reported in [15] for the case of the vortex model (see their Fig. 7). Hence, the application of our simplified model can be justified in this case. Note that despite the overall agreement between the results of modelling and experimental data, the observed rate of temperature decrease at the later times is lower than predicted by the model.

Plots similar to those shown in Figs. 2 and 3, but for the mixtures of ethanol and acetone, are presented in Figs. 4-6. The calcu-

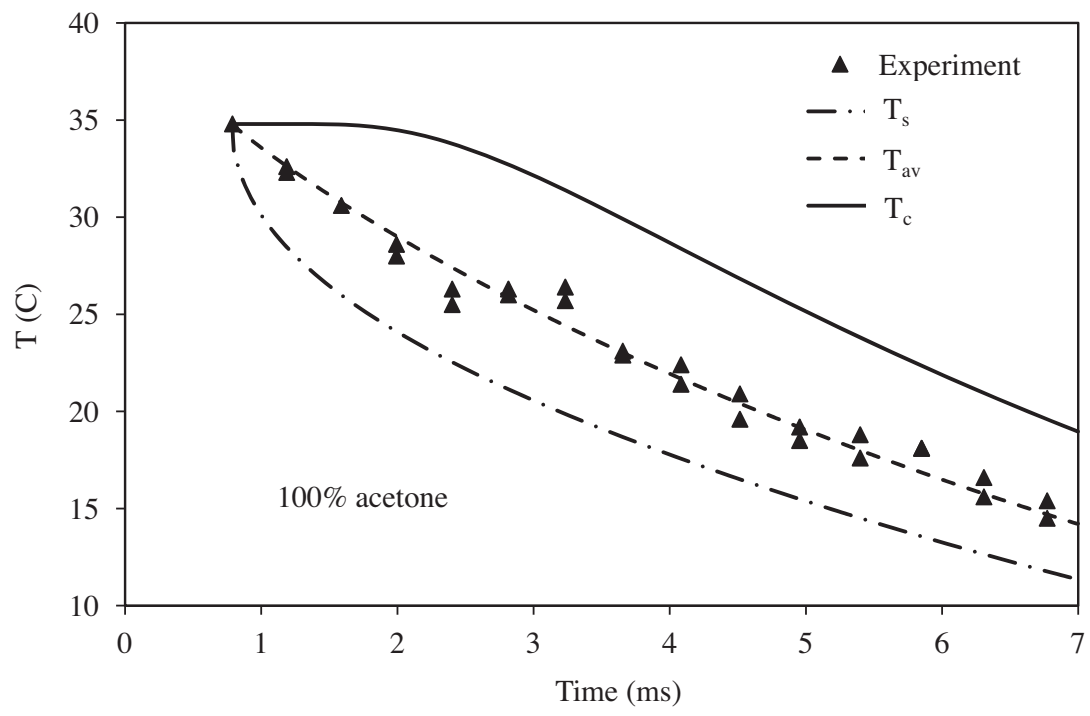

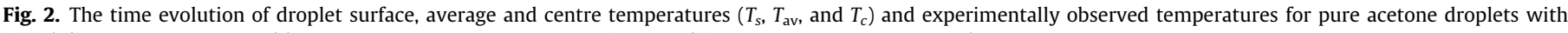
initial diameter $143.4 \mu \mathrm{m}$ and homogeneous temperature $35.1^{\circ} \mathrm{C}$ in an ambient gas at temperature equal to $21.5^{\circ} \mathrm{C}$. 


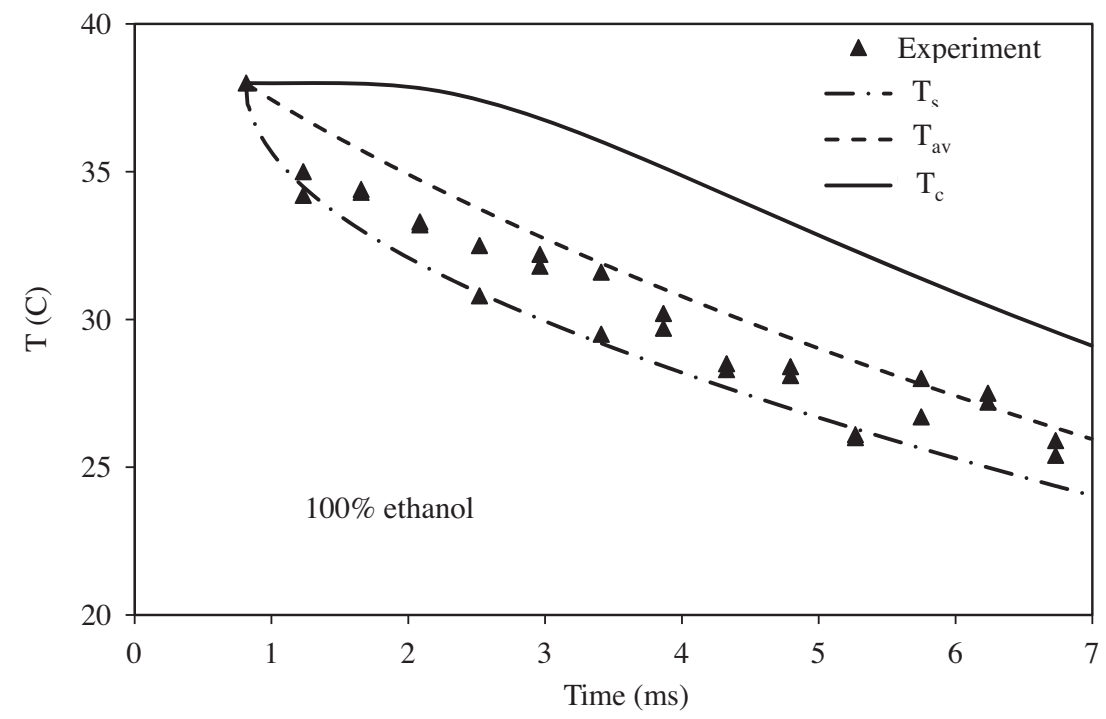

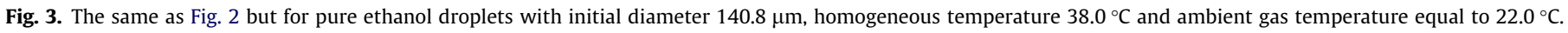

lations were performed for the cases of the ideal mixture $\left(\gamma_{i}=1\right.$ in Eqs. (29) and (30)) and the non-ideal mixture ( $\gamma_{i}$ in Eqs. (29) and (30) was calculated based on Eq. (45)). As can be seen from these figures, in all cases the predictions of the temperatures by the ideal and non-ideal models are noticeably different (by up to several degrees), especially at later times. However, both these models predict about the same trend in the evolution of temperature with time. The ideal model can be used if the prediction errors of several degrees can be tolerated. This seems to be our case where the random errors of the estimates of droplet temperatures seem to be about $2-3^{\circ}$.

In the case of an acetone dominated mixture (25\% ethanol-75\% acetone: see Fig. 4), the agreement between the observed and predicted average droplet temperatures, for both ideal and non-ideal models, is reasonably good, although the scatter of experimental data in this case is more noticeable than in the case of pure acetone (see Fig. 2). In the case of the $50 \%$ ethanol-50\% acetone mixture (see Fig. 5), the experimentally observed temperatures lie between the average and surface temperatures predicted by both ideal and non-ideal models, as in the case of pure ethanol shown in Fig. 3. These temperatures are closer to the average temperatures predicted by the non-ideal model than to those predicted by the ideal model. In the case of the $75 \%$ ethanol-25\% acetone mixture (see Fig. 6), the experimentally observed temperatures lie well below the average temperatures predicted by both ideal and non-ideal models, although they are closer to the average temperatures predicted by the non-ideal model than to those predicted by the ideal model, as in the case of the $50 \%$ ethanol-50\% acetone mixture. The reason for this deviation between the measured and predicted temperatures, which could reach up to $5 \mathrm{~K}$, is not clear to us.

In Fig. 7 we have compared the predictions of our simplified model and the predictions of the vortex model, reported in [15]. As one can see from this figure, the experimental results agree better with the predictions of the average temperature by the vortex model than by our simplified model. It seems, however, that this was achieved by the choice of the lower initial droplet temperature in the vortex model (which could be used as a fitting parameter). If the values of this temperature were taken to be the same, one

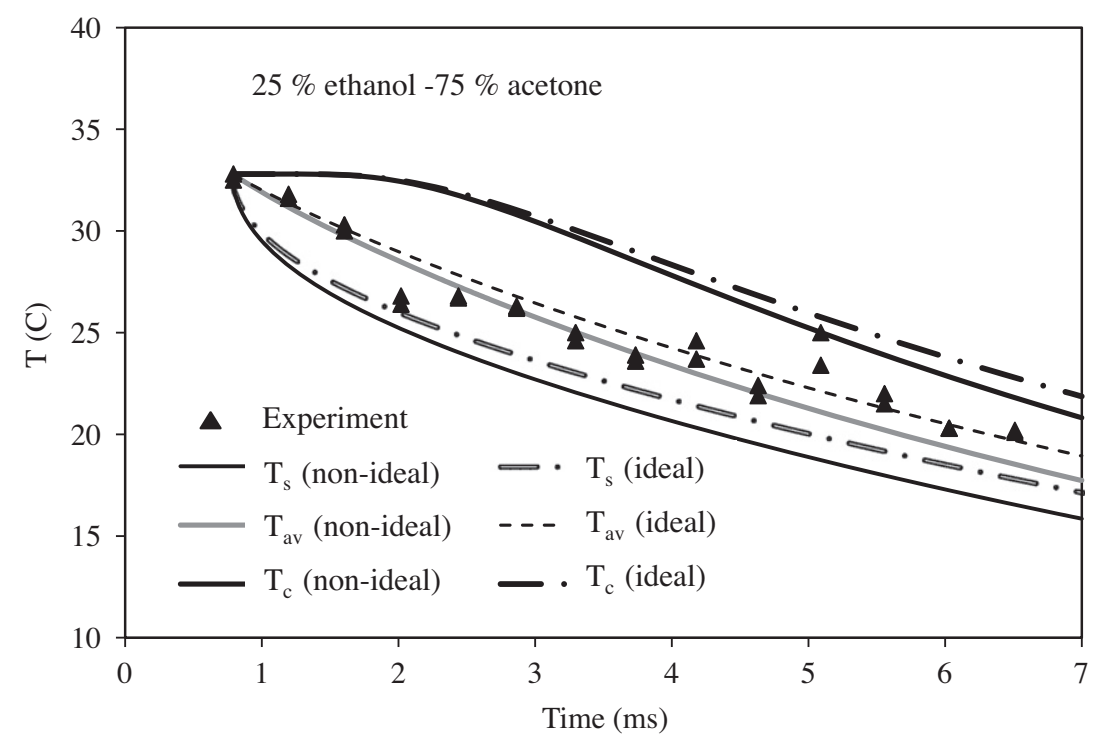

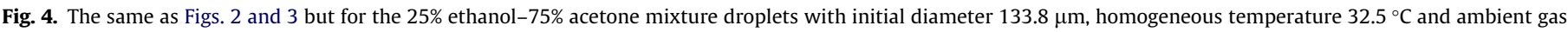
temperature equal to $21.1^{\circ} \mathrm{C}$. The results of calculations based on the ideal $\left(\gamma_{i}=1\right)$ and non-ideal models are presented. 


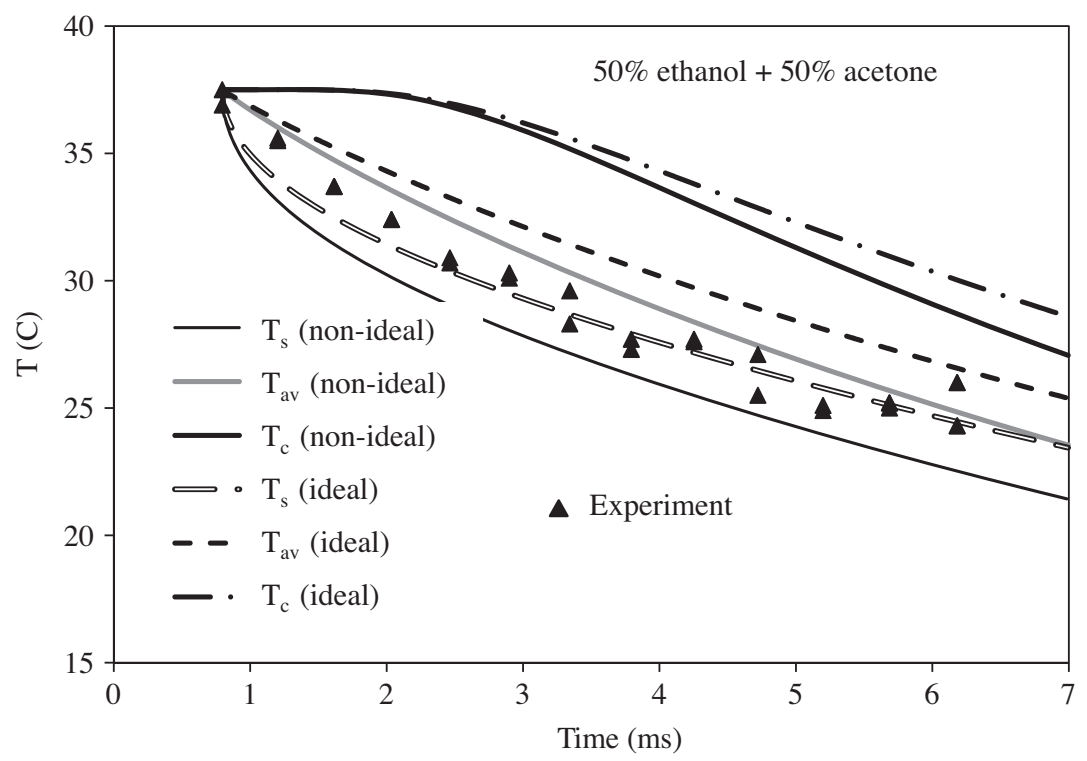

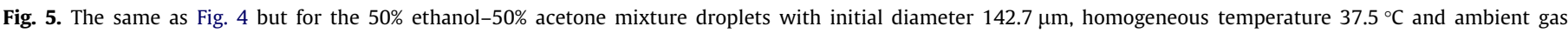
temperature equal to $20.8^{\circ} \mathrm{C}$.

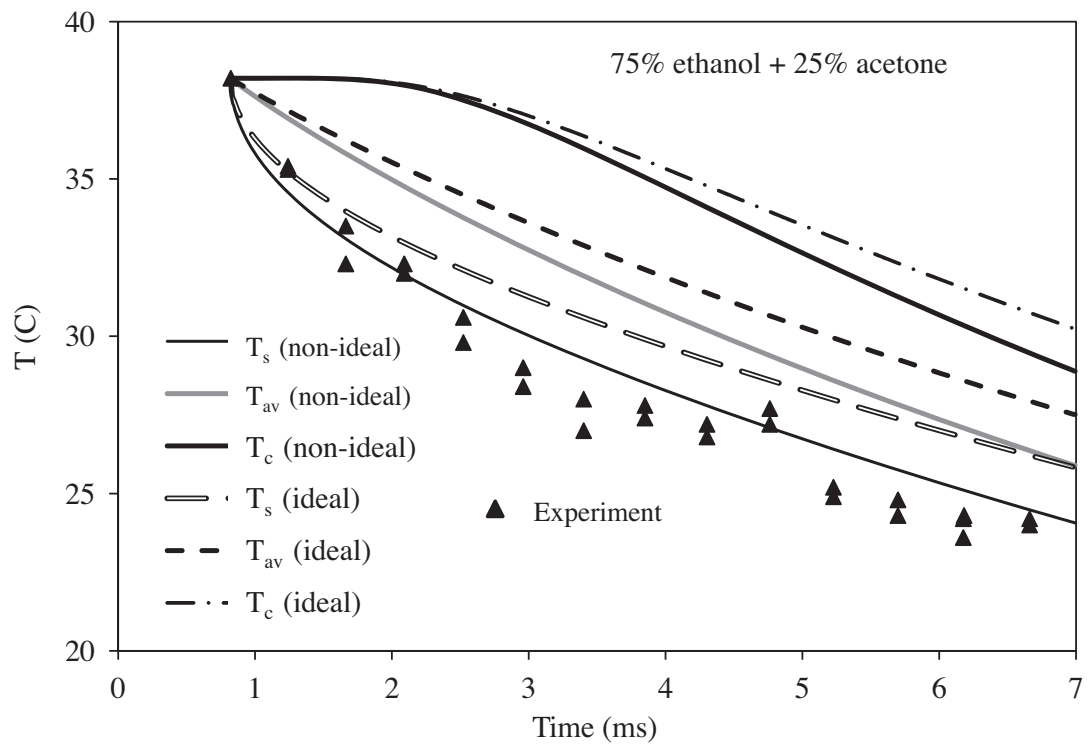

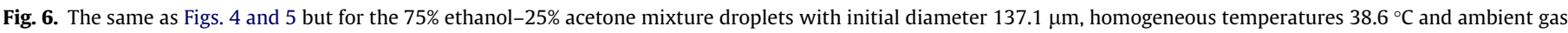
temperature equal to $21.6^{\circ} \mathrm{C}$.

would expect that the predictions of the simplified and vortex models would be very close.

The plots of time evolution of droplet radius for the same mixture as in the case of Figs. 6 and 7, inferred from our simplified model and the numerical results reported in [15], are shown in Fig. 8. As follows from this figure, the vortex model predicts a slightly higher evaporation rate than the simplified model (the difference in predicted radii is less than $0.3 \mu \mathrm{m}$ ). In both cases, the non-ideal model predicts a slightly higher evaporation rate than the ideal one.

\section{Conclusions}

A simplified model for bi-component droplet heating and evaporation is suggested. This model takes into account droplet heating by convection from the ambient gas, the distribution of tempera- ture inside the droplet, diffusion of liquid species inside the droplet, droplet swelling or contraction due to changing average temperature, effects of the non-unity activity coefficient (ideal and non-ideal models) and the effects of interaction between moving droplets due to the finite distance parameter (ratio of the average distance between droplets to their diameter). The effects of recirculation in the moving droplets on heat and species mass diffusion within them are taken into account using the effective thermal conductivity and effective diffusivity models. The previously obtained analytical solution of the transient heat conduction equation has been incorporated in the numerical code alongside the original analytical solution of the species diffusion equation inside droplets.

The predicted time evolutions of surface, average and central droplet temperatures have been compared with the results of direct measurements of droplet average temperatures for the case 


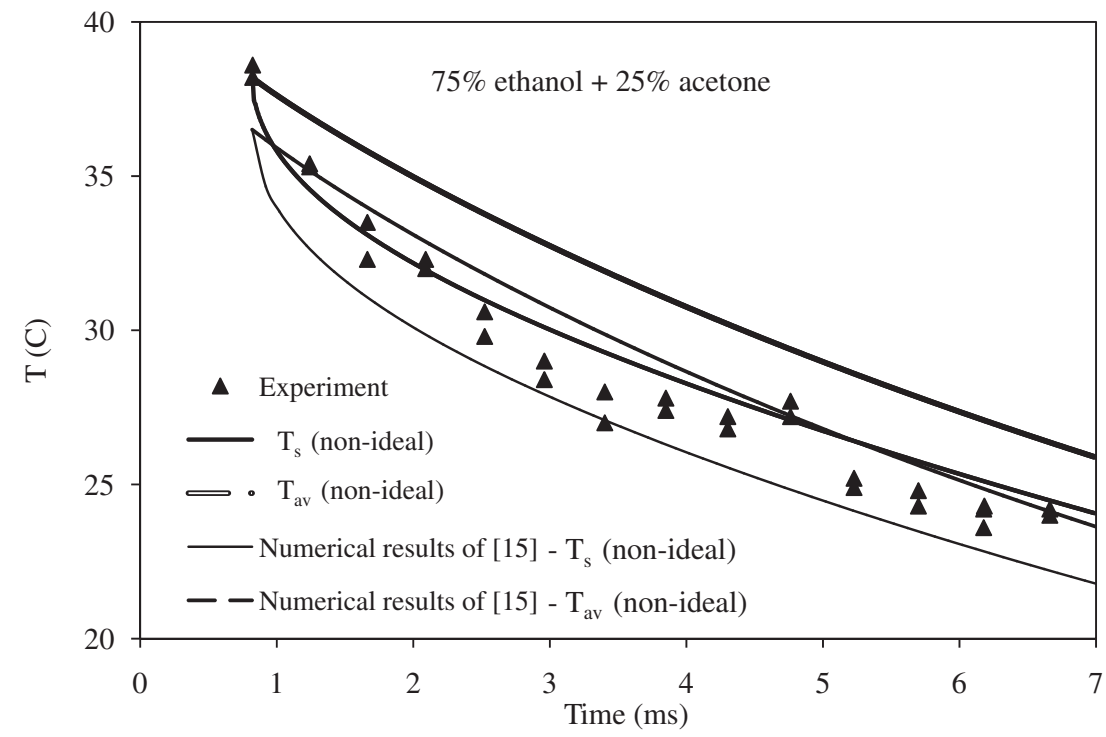

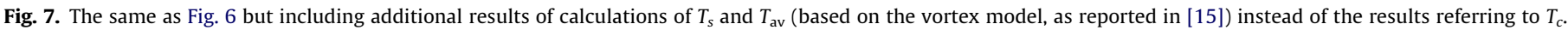

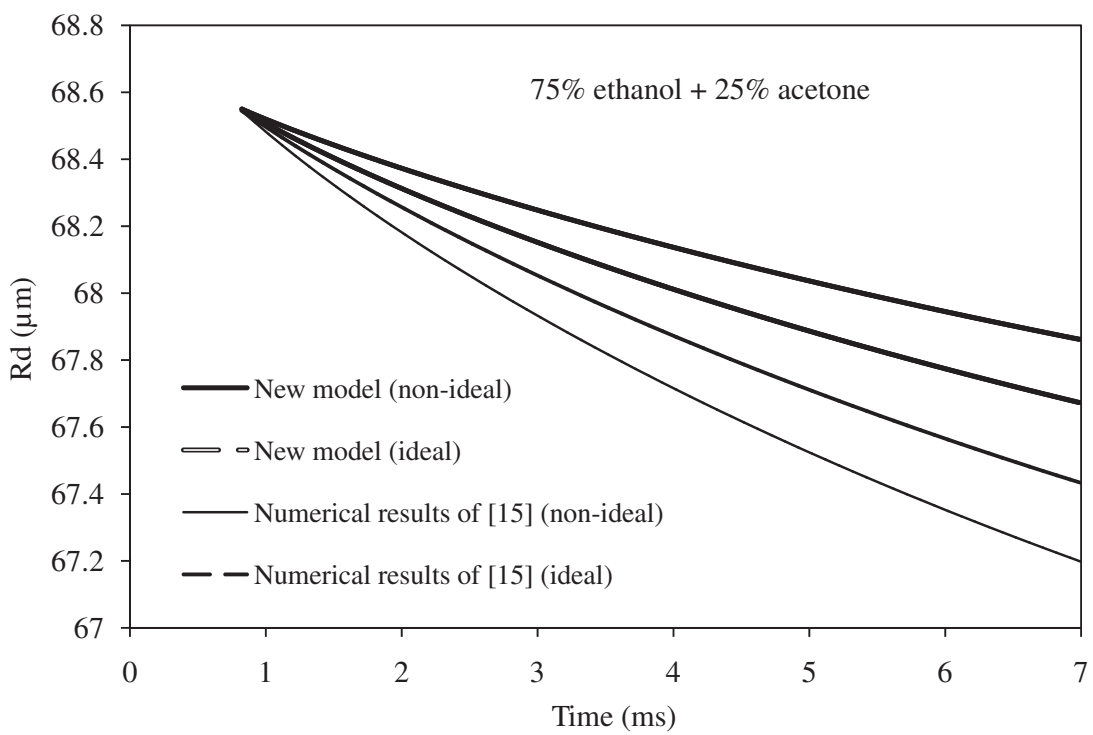

Fig. 8. The plots of $R_{d}$ versus time predicted by the ideal $\left(\gamma_{i}=1\right)$ and non-ideal, simplified and vortex models for the same droplets as in Figs. 6 and 7 .

of various mixtures of ethanol and acetone. There is a general agreement between the predicted and observed average temperatures in the case of pure acetone and acetone-rich mixtures. In the case of ethanol, $50 \%$ ethanol-50\% acetone and $75 \%$ ethanol- $25 \%$ acetone mixture droplets the predicted average droplet temperature was several degrees (up to $5 \mathrm{~K}$ ) higher compared with the observed one. It was shown that the temperatures predicted by the simplified model and the earlier reported vortex model were reasonably close. Also, the temperatures predicted by the ideal and non-ideal models differ by not more that several degrees. This can justify the application of the simplified model with the activity coefficient equal to 1 for the interpretation of the time evolution of temperatures measured with similar errors.

\section{Acknowledgements}

The authors are grateful to the European Regional Development Fund Franco-British INTERREG IVA (Project C5, Reference 4005) and the Royal Society (UK) (Project JP71790) for the financial support of this project.

\section{Appendix A. Transport coefficients for mixtures}

\section{A.1. Liquid thermal conductivity for a mixture}

A number of approximate formulae for thermal conductivity in multi-component liquids are reviewed in [31]. We use the most simple Filippov equation, valid for binary mixtures:

$k_{l}=Y_{1} k_{l 1}+Y_{2} k_{l 2}-0.72 Y_{1} Y_{2}\left|k_{l 1}-k_{l 2}\right|$,

where $Y_{1}$ and $Y_{2}$ are mass fractions for species 1 and $2, k_{l 1}$ and $k_{l 2}$ are thermal conductivities of species 1 and 2 . The thermal conductivities of ethanol $\left(k_{l, \text { eth }}\right)$ and acetone $\left(k_{l, \text { ac }}\right)$ (in $\mathrm{W} /(\mathrm{mK})$ ) were calculated based on the following approximations $[38,39,30]$ : 
$k_{l, \text { eth }}=0.61572-0.24127 \times 10^{-2} \times T+0.31333 \times 10^{-5} \times T^{2}$,

$k_{l, \mathrm{ac}}=0.3133614225-0.8163 \times 10^{-3} \times T+0.1 \times 10^{-5} \times T^{2}$,

where $T$ is in K everywhere.

\section{A.2. Liquid viscosity for a mixture}

A number of approximate formulae for dynamic viscosity of multi-component liquids $\left(\mu_{l}\right)$ are reviewed in [31]. It was shown that the approach suggested by Grunberg and Nissan is the most accurate and convenient in practical applications. In the case of a bi-component liquid, this approach is based on the following equation:

$\ln \mu_{l}=X_{1} \ln \mu_{l 1}+X_{2} \ln \mu_{l 2}-G_{12} X_{1} X_{2}$,

where $X_{1,2}$ are molar fractions of species 1 and 2 . The values of $G_{12}$ depend on the type of species involved [31]. In our case, following [30], we use a simplified version of Eq. (35) assuming that $G_{12}=0$. In this case Eq. (35) is simplified to:

$\mu_{l}=\exp \left[X_{1} \ln \mu_{l 1}+X_{2} \ln \mu_{l 2}\right]$.

The kinematic viscosity is obtained from Eq. (36) by dividing $\mu_{l}$ by

$\rho_{l}=\left(\frac{Y_{1}}{\rho_{l 1}}+\frac{Y_{2}}{\rho_{l 2}}\right)^{-1}$,

where $\rho_{1,2}$ are densities of the components. The specific heat capacity of the mixture $c_{l}$ was estimated as

$c_{l}=Y_{1} c_{l 1}+Y_{2} c_{l 2}$.

The values of $\mu_{l}$ (in Pa s), $\rho_{l}$ (in $\mathrm{kg} / \mathrm{m}^{3}$ ) and $c_{l}$ (in $\mathrm{J} /(\mathrm{kg} \mathrm{K})$ ) for ethanol and acetone were estimated based on the following approximations $[38,39,30]$ :

$\mu_{l, \text { eth }}=10^{\left(\frac{686.64}{T}-5.282\right)}$,

$\mu_{l, \mathrm{ac}}=0.3183313525 \times 10^{-2}-0.1629735179 \times 10^{-4} \times T$

$$
+0.223333 \times 10^{-7} \times T^{2}
$$

$\rho_{l, \mathrm{eth}}=1053.6-0.925 \times T$,

$\rho_{l, \mathrm{ac}}=986.5303588-0.6014966034 \times T-0.2754046133 \times 10^{-3} \times T^{2}$,

$c_{l, \text { eth }}=15039-130.53 \times T+0.4143 \times T^{2}-0.39583 \times T^{3}$,

$c_{l, \mathrm{ac}}=2165.234225-2.963 \times T+0.01 \times T^{2}$.

\section{A.3. Liquid diffusivity for a mixture}

As in the case of liquid thermal conductivity and liquid viscosity, various approximations for liquid diffusivity for a mixture were discussed in [31]. One of the simplest approximations was given by the Sanchez and Clifton formula:

$D_{12}=\left[X_{1} D_{12}^{0}+X_{2} D_{21}^{0}\right](1-\bar{m}+\bar{m} \bar{a})$,

where the parameter $\bar{m}$ is to be found from one mixture datum point, $\bar{a}$ is the thermodynamic factor defined as:

$\bar{a}=\left.\frac{\partial a_{1}}{\partial X_{1}}\right|_{T, p}=\left.\frac{\partial a_{2}}{\partial X_{2}}\right|_{T, p}$,

$a_{1,2}$ are the activities of species 1 and $2, D_{12}^{0}$ and $D_{21}^{0}$ are diffusivities of dilute solute 1 in solvent 2 , and dilute solute 2 in solvent 1 .
Since both $\bar{m}$ and $\bar{a}$ are close to 1, Formula (38) can be simplified to

$D_{12}=X_{1} D_{12}^{0}+X_{2} D_{21}^{0}$.

Among various approximations for $D_{12}^{0}$ and $D_{21}^{0}$ we have chosen the Wilke-Chang approximation given by the following formula:

$D_{A B}^{0}=\frac{7.4 \times 10^{-12} \sqrt{\varphi M_{B}} T}{\mu_{B} V_{A}^{0.6}}$,

where $D_{A B}^{0}$ is the mutual diffusion coefficient of solute $A$ at very low concentrations in solvent $B, \mathrm{~m}^{2} / \mathrm{s}, M_{B}$ is the molar mass of solvent $B$, $\mathrm{kg} / \mathrm{kmol}, T$ temperature in $\mathrm{K}, \mu_{B}$ dynamic viscosity of solvent $B, \mathrm{cP}$ $\left(1 \mathrm{cP}=10^{-3} \mathrm{~kg} \mathrm{~m}^{-1} \mathrm{~s}^{-1}\right), V_{A}$ is the molar volume of solute $A$ at its normal boiling temperature, $\mathrm{cm}^{3} / \mathrm{mol}$ (this can be recalculated from density if required, taking into account the molar mass of the substance), $\varphi$ is the associated factor of solvent $B(\varphi=1.5$ if $B$ is ethanol, $\varphi=1$ if $B$ is acetone).

$V_{A}$ (in $\mathrm{cm}^{3} / \mathrm{mol}$ ) is calculated from the following equation (cf. Eq. 11-4.2 of [31]):

$V_{A}=\left(\sigma_{A} / 1.18\right)^{3}$,

where the Lennard-Jones length $\sigma_{A}$ in A is taken from Table A1.

When calculating $D_{12}$ based on Eq. (38) the values of $X_{1}, X_{2}$ and $T$, averaged over the whole volume of droplets, were used. These parameters were updated at each timestep.

\section{A.4. Gas diffusivity for a mixture} [31]:

The value of $D_{v}$ is estimated from the Wilke and Lee formula

$D_{v}=\frac{\left[3.03-\left(0.98 / M_{v a}^{1 / 2}\right)\right]\left(10^{-7}\right) T^{3 / 2}}{p M_{v a}^{1 / 2} \sigma_{v a}^{2} \Omega_{D}}$,

where $D_{v}$ is in $\mathrm{m}^{2} / \mathrm{s}, T$ is temperature in $\mathrm{K}$,

$M_{v a}=2\left[\left(1 / M_{v}\right)+\left(1 / M_{\mathrm{air}}\right)\right]^{-1}$

$M_{v}$ and $M_{\text {air }}$ are molar masses of vapour and air, respectively, $p$ is pressure in bar, $\sigma_{v a}=\left(\sigma_{v}+\sigma_{a}\right) / 2, \sigma_{v}$ and $\sigma_{a}$ are characteristic Lennard-Jones lengths for vapour and air, respectively, measured in Angstrom (A), $\Omega_{D}$ is the function of the normalised temperature $T^{*}=k_{B} T / \varepsilon_{v a}$ given by Eq. (B6) in [4], $\varepsilon_{v a}=\sqrt{\varepsilon_{v} \varepsilon_{a}}, \varepsilon_{v}$ and $\varepsilon_{a}$ are characteristic Lennard-Jones energies for vapour and air, respectively, $k_{B}$ is the Boltzmann constant. The values of $\sigma_{v, a}$ and $\varepsilon_{v, a} / k_{B}$ for air, ethanol and acetone are given in Table A1 [31]:

In the case of the mixture of vapour components, all input parameters (molar masses and Lennard-Jones parameters) are calculated as molar averaged, taking into account their relative molar concentrations $X_{\text {eth }} /\left(X_{\text {eth }}+X_{\text {acet }}\right)$ and $X_{\text {acet }} /\left(X_{\text {eth }}+X_{\text {acet }}\right)$.

\section{A.5. Gas thermal conductivity and viscosity}

The thermal conductivity $\left(k_{v}\right)$ and dynamic viscosity $\left(\mu_{v}\right)$ for the mixture of air, ethanol vapour and acetone vapour was calculated following the procedure described in [4] (see their Appendix B).

Table A1

The Lennard-Jones lengths and energies for air, ethanol and acetone.

\begin{tabular}{llc}
\hline Substance & $\sigma_{v, a}(\mathrm{~A})$ & $\varepsilon_{v, a} / k_{B}(\mathrm{~K})$ \\
\hline Air & 3.711 & 78.6 \\
Ethanol & 4.530 & 362.6 \\
Acetone & 4.600 & 560.2 \\
\hline
\end{tabular}


The following approximations for $k_{v}$ (in $\mathrm{W} /(\mathrm{m} \mathrm{K})$ ) and $\mu_{v}$ (in Pa s) for ethanol and acetone vapour were used $[38,39,30]$ :

$$
\begin{aligned}
& k_{v, \text { eth }}=1.8037 \times 10^{-3}+7.419 \times 10^{-6} \times T+1.1536 \times 10^{-7} \times T^{2}, \\
& k_{v, \text { ac }}=0.01143468\left(\frac{T}{273.15}\right)^{2-0.3428433650 \times 10^{-6} \times T^{2}}, \\
& \mu_{v, \text { eth }}=-0.19757 \times 10^{-6}+0.29211 \times 10^{-7} \times T,
\end{aligned}
$$$$
\mu_{v, \mathrm{ac}}=-0.1579884447 \times 10^{-5}+0.3090958433 \times 10^{-7} \times T
$$$$
-0.449329 \times 10^{-11} \times T^{2} \text {. }
$$

\section{Appendix B. Activity coefficient for the ethanol-acetone mixtures}

The activity coefficient $\left(\gamma_{i}\right)$ for any liquid mixture is related to the excess of Gibbs free energy per unit mole $\left(G^{E}\right)$ by the following formula [28]:

$\ln \gamma_{i}=\frac{1}{R_{u} T} \frac{\partial\left(n_{l, \text { total }} G^{E}\right)}{\partial n_{l i}}$,

where $R_{u}$ is the universal gas constant, $T$ is temperature in $\mathrm{K}$, $n_{l, \text { total }}=\sum_{i} n_{l i}, n_{l i}$ is the molar concentration of the $i$ th component in the liquid phase.

The following approximation for the ethanol $(i=1)$-acetone $(i=2)$ mixture, based on fitting experimental data, was suggested [29]:

$n_{l, \text { total }} G^{E}=R_{u} T \frac{n_{l 1} n_{l 2}}{n_{l 1}+n_{l 2}}\left[\frac{\alpha n_{l 2}}{n_{l 1}+n_{l 2}}+\frac{\beta n_{l 1}}{n_{l 1}+n_{l 2}}-\frac{\delta n_{l 1} n_{l 2}}{\left(n_{l 1}+n_{l 2}\right)^{2}}\right]$,

where

$\alpha=\frac{546.3}{T}-0.9897$,
$\beta=\frac{543.3}{T}-0.9483$
$\delta=\frac{15.63}{T}+0.0759$.

Having substituted (44) into (43) the following expressions for $\gamma_{1}=\gamma_{\text {eth }}$ and $\gamma_{2}=\gamma_{\mathrm{ac}}$ are obtained:

$\left.\gamma_{1}=\exp \left[X_{l 2}^{2}\left[\alpha+2(\beta-\alpha-\delta) X_{l 1}+3 \delta X_{l 1}^{2}\right]\right]\right\}$.

$\left.\gamma_{2}=\exp \left[X_{l 1}^{2}\left[\beta+2(\alpha-\beta-\delta) X_{l 2}+3 \delta X_{l 2}^{2}\right]\right]\right\}$.

Expressions (45) were used in Eq. (29).

\section{References}

[1] S.S. Sazhin, P.A. Krutitskii, A conduction model for transient heating of fuel droplets, in: H.G.W. Begehre, R.P. Gilbert, M.W. Wong (Eds.), Progress in analysis, Proceedings of the 3rd International ISAAC (International Society for Analysis, Applications and Computations) Congress (August 20-25, 2001, Berlin), vol. II, World Scientific, Singapore, 2003, pp. 1231-1239.

[2] S.S. Sazhin, P.A. Krutitskii, W.A. Abdelghaffar, S.V. Mikhalovsky, S.T. Meikle, M.R. Heikal, Transient heating of diesel fuel droplets, Int. J. Heat Mass Transfer 47 (2004) 3327-3340.

[3] S.S. Sazhin, W.A. Abdelghaffar, P.A. Krutitskii, E.M. Sazhina, M.R. Heikal, New approaches to numerical modelling of droplet transient heating and evaporation, Int. J. Heat Mass Transfer 48 (2005) 4215-4228.

[4] S.S. Sazhin, T. Kristyadi, W.A. Abdelghaffar, M.R. Heikal, Models for fuel droplet heating and evaporation: comparative analysis, Fuel 85 (2006) 1613-1630.

[5] S.S. Sazhin, Advanced models of fuel droplet heating and evaporation, Prog. Energy Combust. Sci. 32 (2006) 162-214.

[6] C. Bertoli, M. Migliaccio, A finite conductivity model for diesel spray evaporation computations, Int. J. Heat Fluid Flow 20 (1999) 552-561.
[7] C. Maqua, G. Castanet, F. Grisch, F. Lemoine, T. Kristyadi, S.S. Sazhin, Monodisperse droplet heating and evaporation: experimental study and modelling, Int. J. Heat Mass Transfer 51 (2008) 3932-3945.

[8] T. Kristyadi, V. Deprédurand, G. Castanet, F. Lemoine, S.S. Sazhin, A. Elwardany, E.M. Sazhina, M.R. Heikal, Monodisperse monocomponent fuel droplet heating and evaporation, Fuel 89 (2010), in press.

[9] G.M. Faeth, Evaporation and combustion of sprays, Prog. Energy Combust. Sci. 9 (1983) $1-76$.

[10] A.Y. Tong, W.A. Sirignano, Multicomponent transient droplet vaporization with internal circulation: integral equation formulation, Numer. Heat Transfer 10 (1986) 253-278.

[11] G. Continillo, W.A. Sirignano, Unsteady, spherically-symmetric flame propagation through multicomponent fuel spray clouds, in: G. Angelino, L. De Luca, W.A. Sirignano (Eds.), Modern Research Topics in Aerospace Propulsion, Springer-Verlag, 1991, pp. 173-198.

[12] M. Klingsporn, U. Renz, Vaporization of a binary unsteady spray at high temperature and high pressure, Int. J. Heat Mass Transfer 37 (Suppl. 1) (1994) 265-272.

[13] P.L.C. Lage, C.M. Hackenberg, R.H. Rangel, Nonideal vaporization of dilating binary droplets with radiation absorption, Combust. Flame 101 (1995) 3644.

[14] D.J. Torres, P.J. O’Rourke, A.A. Amsden, Efficient multi-component fuel algorithm, Combust. Theory Model. 7 (2003) 67-86.

[15] C. Maqua, G. Castanet, F. Lemoine, Bi-component droplets evaporation: temperature measurements and modelling, Fuel 87 (2008) 2932-2942.

[16] J. Tamim, W.L.H. Hallett, Continuous thermodynamics model for multicomponent vaporization, Chem. Eng. Sci. 50 (1995) 2933-2942.

[17] A.M. Lippert, R.D. Reitz, Modelling of Multicomponent Fuels using Continuous Distributions with Application to Droplet Evaporation and Sprays, SAE Technical Paper 972882, 1997.

[18] G.-S. Zhu, R.D. Reitz, A model for high-pressure vaporization of droplets of complex liquid mixture using continuous thermodynamics, Int. J. Heat Mass Transfer 45 (2002) 495-507.

[19] M. Burger, R. Schmehl, K. Prommersberger, O. Schäfer, R. Koch, S. Wittig, Droplet evaporation modelling by the distillation curve model: accounting for kerosene fuel and elevated pressures, Int. J. Heat Mass Transfer 46 (2003) 4403-4412.

[20] C. Laurent, Dévelopment et validation de modèles d'évaporation multicomposant, Thèse de l'Institut Supériour de l'Aéronautique et de l'Espace de Toulouse, 2008.

[21] H.S. Carslaw, J.C. Jaeger, Conduction of Heat in Solids, Clarendon Press, Oxford, 1986.

[22] E.M. Kartashov, Analytical Methods in Heat Transfer Theory in Solids, Vysshaya Shkola, Moscow, 2001 (in Russian).

[23] B. Abramzon, W.A. Sirignano, Droplet vaporization model for spray combustion calculations, Int. J. Heat Mass Transfer 32 (1989) 1605-1618.

[24] B. Abramzon, S. Sazhin, Droplet vaporization model in the presence of thermal radiation, Int. J. Heat Mass Transfer 48 (2005) 1868-1873.

[25] B. Abramzon, S. Sazhin, Convective vaporization of fuel droplets with thermal radiation absorption, Fuel 85 (2006) 32-46.

[26] W.A. Sirignano, Fluid Dynamics and Transport of Droplets and Sprays, Cambridge University Press, Cambridge, 1999.

[27] J.-P. Delplanque, R.H. Rangel, W.A. Sirignano, Liquid-waste incineration in a parallel-stream configuration: effect of auxiliary fuel, Progress in Aeronautics Astronautics 132 (1991) 164-184.

[28] P. Atkins, J. de Paula, Atkins' Physical Chemistry, seventh ed., Oxford University Press, 2002.

[29] S.W. Campbell, R.A. Willsak, G. Thodos, Vapor-liquid equilibrium measurements for the ethanol-acetone system at 372.7, 397.7 and $422.6 \mathrm{~K}$, J. Chem. Eng. Data 32 (1987) 357-362.

[30] C. Maqua, Contribution à la compréhension de l'évaporation de gouttes de combustible bi-composant à l'aide de méthodes optique, Thèse, NancyUniversité, 2007.

[31] B.E. Polling, J.M. Prausnitz, J.P. O'Connell, The Properties of Gases and Liquids, McGraw-Hill, 2000.

[32] C. Maqua, G. Castanet, N. Doué, G. Lavergne, F. Lemoine, Temperature measurements of binary droplets using three color laser-induced fluorescence, Exp. Fluids 40 (2006) 786797.

[33] G. Castanet, A. Delconte, F. Lemoine, L. Mees, G. Grehan, Evaluation of temperature gradients within combusting droplets in linear stream using two colors laser-induced fluorescence, Exp. Fluids 39 (2005) 431-440.

[34] K.M. Watson, Thermodynamics of the liquid state, Ind. Eng. Chem. 35 (1943) 398-406.

[35] G. Castanet, C. Maqua, M. Orain, F. Grisch, F. Lemoine, Investigation of heat and mass transfer between the two phases of an evaporating droplet stream using laser-induced fluorescence techniques: comparison with modelling, Int. J. Heat Mass Transfer 50 (2007) 3670-3683.

[36] L.A. Dombrovsky, S.S. Sazhin, A parabolic temperature profile model for heating of droplets, ASME J. Heat Transfer 125 (2003) 535-537.

[37] L.A. Dombrovsky, S.S. Sazhin, A simplified non-isothermal model for droplet heating and evaporation, Int. Commun. Heat Mass Transfer 30 (2003) 787796.

[38] R.C. Reid, J.M. Prausnitz, T.K. Sherwood, The Properties of Gases and Liquids, third ed., Mc Graw Hill, 1977.

[39] R.C. Reid, J.M. Prausnitz, B.E. Poling, The Properties of Gases and Liquids, fourth ed., Mc Graw Hill, 1987. 